\section{Designer Genes}

The title of this molecular biology site may attract a few poor spellers looking for Calvin Klein, but they'll almost certainly be disappointed. On the other hand, molecular biologists who'd like to reverse translate the one letter protein sequence "c-a-l-v-i-n-k-l-e-i-n" won't be.
Home to a simple, yet powerful set of software applications for basic molecular biological analyses, Gene Design is aimed at researchers who want to manipulate genes, but don't want to pay for a commercial package to do so. The site's online tools allow users to alter codons in a sequence without disturbing the reading frame, insert or remove silent mutations, and perform basic analyses. These include open reading frame identification, restriction enzyme analysis/identification, and oligonucleotide design.

slam.bs.jhmi.edu/gd

\section{Out of the Muck}

A staple of any biology laboratory class involving a microscope and a bit of murky pond water, the euglenoids are protists known for their extensive endomembrane system. With a full complement of organelles, including an extensive endoplasmic reticulum

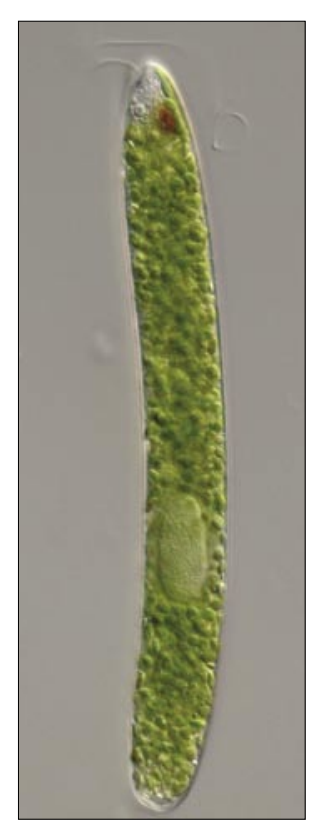
and a pellicular cell surface unique among eukaryotes, these tiny dynamos are not just fun to watch swimming around under the 'scope, they're also lab curiosities. Witness their three membrane chloroplasts or the large dictyosomes within their Golgi apparati. Richard Triemer's "The Euglenoid Project" is an NSF-funded effort between academic institutions, botanical gardens, water-related institutes, and museums to help spread the word and advance research on these little green bundles. From movies that will bring back memories of your first biology class to phylogenetic sequence alignments, The Euglenoid Project has something for almost anyone fascinated by living systems.

\section{bio.rutgers.edu/euglena}

\section{Gastropodally Yours}

Pop quiz-which mollusk genus has more than 3000 species, contains organisms that make potent conotoxins that are the substance of 80 patents, and is more diverse than any other marine gastropod? Give yourself a gold star if you guessed Conus. If you didn't, consider entering the URL below in your browser. There, at The Conus Biodiversity site, you'll find a cornucopia of information about these organisms that double their number of species every six million years. Hosted by Alan Kohn at the University of Washington, the pages provide an amazing collection of pictures in the Type Gallery, videos of Conus species in action, and an extensive organism catalogue.

\section{biology.burke.washington.edu/ conus}

\section{Not Sappy}

A K-16 educational resource on all things botanical, Science and Plants for Schools (SAPS), lists three main aims. The common theme of all of them is stated in the last one-to "interest young people in plants and molecular biology." The target audience of SAPS is not primarily students, but instead teachers, for whom there is a lot of material to revel in. They will welcome kits (including PCR) that provide classroom exercises, as well as downloadable books, workshops, and a free newsletter. Young scholars aren't totally left out of the picture, thanks to a Question/Answer section where experts provide feedback to their inquiries. If you've ever wondered about problems ranging from the causes of root callusing to the nutritional needs of fast plants, you too may benefit from this informative site.

\section{www-saps.plantsci.cam.ac.uk}

\section{Public Domain}

The free exchange of ideas is a foundation of modern science, but traditional publishing models have, through increasing subscription prices, provided a barrier, at least by some perspectives. Might the open access movement, which has revolutionized software design, provide a better model for the distribution of scientific information? At the Public Library of Science (PLoS), the answer is a resounding "Yes!" With seven peer-reviewed journals in the biological sciences and a policy that allows for unrestricted use, distribution, and reproduction in any medium, as long as the original work is cited, PLoS is both popular and positioned to grow.

\section{www.plos.org/index.php}

- Kevin Ahern - Please send web site recommendations to ahernk@orst.edu 